\title{
ON A PROBLEM OF MAHLER IN THE GEOMETRY OF NUMBERS
}

\author{
A. C. WOODS
}

\begin{abstract}
If $K$ is a convex body in $R_{n}$ and $K(t)$ is that part of $K$ which satisfies $\left|x_{n}\right| \leqq t$, Mahler [2] has shown that $\Delta K(t) / t$ is a decreasing function of $t$, where $\Delta(K)$ is the critical determinant of $K$. We generalise Mahler's result in a way different from that conjectured by him.
\end{abstract}

1. Let $K$ be a closed, convex body in euclidean $n$-space $R_{n}$, symmetric in the origin 0 . For a real and positive number $t$, denote by $K(t)$ that part of $K$ which satisfies $\left|x_{n}\right| \leqq t$, where $x_{n}$ is the $n$th coordinate of a fixed coordinate system. If $V(K)$ denotes the volume of $K$, it was pointed out by Mahler [2] that $V(K(t)) / t$ is a monotone decreasing function of $t$, and he conjectured the same holds true for the critical determinant of $K$. This number is defined as the infimum of the determinants of those lattices which do not contain an inner point of $K$, apart from 0 . Mahler proved his conjecture for $n=2$. Bambah [1] showed that the same result holds true for the covering constant of $K$. This number is defined as the supremum of the determinants of those lattices $L$, for which the collection $X+K$, $X \in L$, covers $R_{n}$. The object of this note is to show these results have a natural extension to $n$ dimensions, along different lines to those conjectured by Mahler, the proof of which depends solely on the simple affine properties of the constants involved.

2. Let $m \leqq n$ be a positive integer, and let $C$ denote any set in $R_{m}$. We assume that $R_{m}$ is embedded in $R_{n}$ in the usual manner. For a real and positive number $t$, we denote by $K(t)$ the set given by $K(t)=K \cap\left(t C \times R_{n-m}\right)$, where $t C \times R_{n-m}=\left\{\left(x_{1}, \cdots, x_{n}\right) \mid\left(x_{1}, \cdots, x_{m}\right) \in t C\right\}$. We restrict the set $C$, throughout, to be such that $K(t)$ has Jordan content for all relevant $t$.

THEOREM 1. $V(K(t)) / t^{m}$ is a monotone decreasing function of $t$.

Let $F$ be a real-valued function of $K$ such that

(i) $F(T(K))=|\operatorname{det} T| F(K)$ for every nonsingular linear transformation $T$,

(ii) if $K_{1} \subset K_{2}$, then $F\left(K_{1}\right) \leqq F\left(K_{2}\right)$.

THEOREM 2. If $m=n-1$, then $F(K(t)) / t^{n-1}$ is a monotone decreasing function of $t$.

Received by the editors May 14, 1972.

AMS (MOS) subject classifications (1969). Primary 1025.

(c) American Mathematical Society 1973 
Theorem 2 represents an extension of the results of Bambah and Mahler to higher dimensions, since the critical determinant and covering constant both satisfy (i) and (ii).

3. Proof of Theorem 1. Let $0<t<t^{\prime}$, and let $T$ denote the linear transformation given by $x_{i} \rightarrow t^{\prime} x_{i} / t$, for $i=1, \cdots, m$, so that $\operatorname{det} T=$ $\left(t^{\prime} \mid t\right)^{m}$. Now

$$
V\left(K\left(t^{\prime}\right)\right)=\int_{R_{m}} V_{1}\left(x_{1}, \cdots, x_{m}\right) d x_{1} \cdots d x_{m},
$$

and

$$
V(T(K(t)))=\int_{R_{m}} V_{2}\left(x_{1}, \cdots, x_{m}\right) d x_{1} \cdots d x_{m}
$$

where $V_{1}\left(x_{1}, \cdots, x_{m}\right)$ is the $n-m$ dimensional volume of the set $S_{1}$,

$$
S_{1}=\left\{\left(y_{1}, \cdots, y_{n}\right) \in K\left(t^{\prime}\right) \mid y_{i}=x_{i} \text { for } i=1, \cdots, m\right\} ;
$$

and $V_{2}\left(x_{1}, \cdots, x_{m}\right)$ is the $n-m$ dimensional volume of the set $S_{2}$,

$$
S_{2}=\left\{\left(y_{1}, \cdots, y_{n}\right) \in T(K(t)) \mid y_{i}=x_{i} \text { for } i=1, \cdots, m\right\} .
$$

Theorem 2 will follow if we can show $V\left(S_{1}\right) \leqq V\left(S_{2}\right)$, for all points $\left(x_{1}, \cdots, x_{m}\right) \in R_{m}$. The set $T^{-1}\left(S_{2}\right)$ is a translation of $S_{2}$, and so has volume $V\left(S_{2}\right)$. Further, it is contained in $K(t)$. Let $V\left(x_{1}, \cdots, x_{m}\right)$ denote the $n-m$ dimensional volume of the set

$$
\left\{\left(y_{1}, \cdots, y_{n}\right) \in K \mid y_{i}=x_{i} \text { for } i=1, \cdots, m\right\} .
$$

As $K$ is symmetric in $0, V\left(x_{1}, \cdots, x_{m}\right)=V\left(-x_{1}, \cdots,-x_{m}\right)$. Therefore, by the Brunn-Minkowski theorem,

$$
V(0, \cdots, 0) \geqq V\left(x_{1}, \cdots, x_{m}\right) .
$$

There is clearly nothing to prove if $S_{1}$ is the empty set, so we may assume that $S_{1}$ is not empty, from which it follows

and

$$
S_{1}=\left\{\left(y_{1}, \cdots, y_{n}\right) \in K \mid y_{i}=x_{i} \text { for } i=1, \cdots, m\right\},
$$

$$
T^{-1}\left(S_{2}\right)=\left\{\left(y_{1}, \cdots, y_{n}\right) \in K \mid y_{i}=t x_{i} / t^{\prime} \text { for } i=1, \cdots, m\right\} .
$$

If $S(0, \cdots, 0)$ denotes the section of $K$ at the origin, namely

$$
S(0, \cdots, 0)=\left\{\left(y_{1}, \cdots, y_{n}\right) \in K \mid y_{i}=0 \text { for } i=1, \cdots, m\right\},
$$

then, applying the Brunn-Minkowski theorem to the sets $S(0, \cdots, 0)$, $T^{-1}\left(S_{2}\right)$ and $S_{1}$ and using the fact already established that $V(0, \cdots, 0) \geqq$ $V\left(S_{1}\right)$, we obtain $V\left(T^{-1}\left(S_{2}\right)\right) \geqq V\left(S_{1}\right)$ and Theorem 1 follows. 
4. Proof of Theorem 2. Let $P$ denote the tangent plane to $K$, at that point of the boundary of $K$ for which $x_{1}=\cdots=x_{n-1}=0, x_{n}=a>0$, say. As the quantities in the theorem are unchanged by linear transformations of determinant 1 that leave the first $n-1$ coordinates fixed, we may assume, after application of such a transformation if necessary, that $P$ is given by $x_{n}=a$. Let $0<t<t^{\prime}$ and denote by $T$ the linear transformation given by

$$
x_{i} \rightarrow t^{\prime} x_{i} / t, \text { for } i=1, \cdots, n-1 .
$$

If we can show that

(iii) $K\left(t^{\prime}\right) \subset T(K(t))$,

then by (i) and (ii),

$$
F\left(K\left(t^{\prime}\right)\right) \leqq F(T(K(t)))=\left(t^{\prime} / t\right)^{n-1} F(K(t))
$$

and the theorem is proved.

We assert that (iii) is true, for let $X \in K\left(t^{\prime}\right)$. We claim that the point $T^{-1}(X)$ is in $K$ and therefore also in $K(t)$. Otherwise, with $X=\left(x_{1}, \cdots, x_{n}\right)$, $T^{-1}(X)=\left(t x_{1} / t^{\prime}, \cdots, t x_{n-1} / t^{\prime}, x_{n}\right)$ is not in $K$ and, since $0<t / t^{\prime}<1$, so $\left(0, \cdots, 0, x_{n}\right)$ is not in $K$. However $P$ being a tacplane to $K$ implies $\left|x_{n}\right|>a$, from which it follows that $X \notin K$, which is impossible. This contradiction proves Theorem 2 .

\section{REFERENCES}

1. R. P. Bambah, An analogue of a problem of Mahler, Res. Bull. Panjab Univ. No. 109 (1957), 299-302. MR 20 \#3509.

2. K. Mahler, On a problem in the geometry of numbers, Roma Ist. Naz. Alta Mat. Rend. Mat. e Appl. (5) 14 (1954), 38-41. MR 16, 802.

Department of Mathematics, Ohio State University, Columbus, Ohio 43210 